\title{
Semantic video annotation for accessible resources in flipped classrooms
}

\author{
Ilaria Torre \\ Department of Computer Science, Bioengineering, \\ Robotics and Systems Engineering \\ University of Genoa, Italy \\ ilaria.torre@unige.it
}

\author{
Gianni Vercelli \\ Department of Computer Science, Bioengineering, \\ Robotics and Systems Engineering \\ University of Genoa, Italy \\ gianni.vercelli@unige.it
}

\begin{abstract}
In this paper we present a project for applying a framework for accessibility to educational activities in flipped classrooms by exploiting semantic video annotation. The framework exploits semantic and adaptive technologies and is aimed to support accessibility to physical real-world things.
\end{abstract}

Keywords: Internet of Things, accessibility, semantic video annotation, user adapted interaction, technology-enhanced learning, Liked Data.

\section{INTRODUCTION}

The advent of the Internet of Things (IoT) and the Web of Things (WoT) has opened new opportunities for people with special needs [1]. The Perception Layer of the IoT is able to identify objects and gather information from the environment, the Network layer transmits information obtained from the Perception layer and the Application layer is a set of services that take as input the data gathered from the Perception layer to satisfy the needs of the users.

In this paper we present a framework for accessibility in the WoT and a project for applying it within educational environments. The proposal is inspired on Linked Data principles, and uses Semantic Video Annotation to support learners with special needs in flipped classrooms.

Recent literature on flipped or inverted classrooms propose to use/realize videos and to record lectures so students can view them out of class when they prefer. This asynchronous approach frees up in class time for learning activities [2], including exercises, laboratory experiments, document analysis or speech presentation. Video annotation in online and flipped classroom is proposed as a mean to improve learner engagement [3, 4], critical reflection [5] and learning performance [6]. Moreover it has been used to support visually impaired people [7]. In this paper, we describe how the WoT and the Semantic Web technologies can be fruitfully adopted together with video annotation tools - to support students with special needs.

The first part of the paper (Section 2) describes the framework for accessibility, while the second part (Section 3) presents the use of semantic video annotation in flipped classrooms.

\section{ACCESSIBILITY FRAMEWORK}

\section{A. Goals and approach}

Accessibility is the term used to indicate whether an object can be used by people of all abilities and disabilities [8]. It is a multi-faceted concept since accessibility may concern real world things and environments as well as web pages, software applications and ICT devices [9]. Accessibility may concern physical and cognitive disability, but also may include logical barriers. For example, an object is not accessible for a user that does not know how to use it but will become accessible after instructing her; or an application form that is not accessible to a user who does not comprehend its language, will become accessible if a proper translation is provided.

The WoT enriches everyday physical things by linking them to their digital counterpart using HTTP standards. Thus, we obtain augmented cyber-physical things $[10,11]$ that can be accessed and used in different ways, by exploiting their digital or their physical side. As a consequence, even if physical objects are not natively accessible, they can become accessible by means of a software layer, or an application, that adapts access and methods of interaction with them. While physical objects cannot be made accessible for everyone, virtual objects connected to physical objects can [12]. For instance, a physical dictionary or a calculator can be not accessible to visually impaired students. However, the accessibility to these realworld objects can be achieved by equipping the dictionary or calculator with a software layer, such as a smartphone application, that provides the information using the adequate modality: audio in case of blindness or simply larger text in case of impaired sight. Hence, if the application is accessible, the dictionary or calculator will become accessible as well. Similarly, if a scene in the environment cannot be perceived by a visually impaired person, a video recording annotated with scene descriptions may enable the subject to understand it.

Notice that the vast consumer electronics market is already filled with smart devices that can be accessed and controlled remotely via apps (smart fridges, thermostats, heart rate monitors, etc.). The approach that we present here, goes in this direction, but enhances accessibility as its main objective and exploits semantic and adaptation technologies, as discussed in 
related works $[13,14,15,16]$. The accessibility framework is funded on three main blocks that can be seen as actions:

1. Enriching physical objects with semantic annotations (the application scenario that will be presented in the following is focused on semantic video annotations);

2. Matching data about the real-world object features against user needs, preferences and current environment (this may require to catch, profile and annotate the user and the usage context);

3. Exploiting adaptive techniques in order to adapt the webbased counterpart of the physical object to make it accessible to different kinds of users in different conditions.

We use Linked Data (LD) as semantic annotation paradigm because of its suitability to foster the integration of heterogeneous data and their connection, sharing and reuse. This is a great improvement with respect to current smart IoT devices in the market since it allows to exploit the available data on the Web about the physical object, the user needs/preferences and especially it allows to share growing knowledge about accessibility requirements. Thus, the framework combines the new approaches based on WoT and LD and exploits adaptation techniques to adapt the interaction of the virtual side of the augmented physical object to make the physical object accessible.

\section{B. Background}

The integration of computational and physical elements, especially when complemented with intelligent mechanisms, has broadened the potential of cyber-physical systems in several areas. WoT cyber-physical objects have been developed to cope with problems including intervention, coordination and augmentation of human capabilities (e.g., healthcare monitoring and delivery). WoT cyber-physical objects can be physical objects with embedded sensors and processing capabilities, but they can also be everyday artifacts (e.g. books, goods, shop shelves, desks) with attached tokens linked to a virtual counterpart on the Web; users access this virtual counterpart by scanning the attached token (e.g., QR code, RFID tags) and by getting information about its location (URI). Smart objects can also embed tiny Web servers which make it possible to communicate to such objects using HTTP standards and also to invoke services provided by these objects.

While previous research studies dealing with accessibility and ICT were most focused on making devices, software and platforms accessible, current projects exploit ICT to offer augmented services. Several FP7 European Projects have worked on providing frameworks and ontologies for accessibility - e.g., Open Accessibility Everywhere (www.aegis-project.eu), Accessibility Assessment Simulation Environment (www.accessible-eu.org), OASIS (oasisproject.eu) and Cloud 4all (cloud4all.info).

Our approach follows a similar direction, but slightly differs from them since it is specifically focused on using ICT to make current physical objects accessible, while the mentioned projects are more focused on enhancing accessibility of digital devices and web applications. Thus our model can exploit the results of these projects (including the ontologies they have defined, in particular ACCESSIBLE and AEGIS) but emphasizes the use of WoT and the use of LD to connect heterogeneous data about accessibility features.

Several applications have been designed to solve accessibility problems. The latest approaches, methods and tools are collected in [17]. However, the real problem when dealing with assistive technologies is that impairments are heterogeneous and often a subject has more disabilities together. Also limiting the analysis to people, and in particular students, with visual impairments, researchers describe the complexity of exploiting assistive technologies since the combination of different impairments and the different levels of impairment influence the way each technological support is experienced [18].

In this scenario, the possibility of dynamically identifying the kind of disability and adapting the virtual side of the physical object becomes a critical challenge. Adaptation is the core of the GPII (gpii.net) project for a global infrastructure for accessibility and the focus of a semantic framework for assistive technology within the Cloud4all FP7 project [19]. This framework is designed to support user interface adaptation to different assistive technologies and configurations. However, also these projects are focused to make digital devices more accessible, while our objective is to exploit WoT and semantic adaptive technologies to make physical objects more accessible.

\section{WoT object annotation}

Building a digital representation of a physical object is the first step to virtualize it. This process associates each object to its digital representation, described in [20] as Digital Object Memory (DOM), which can be passive or active and can be used for different purposes, ranging from storing temporary data obtained by sensors to storing and representing complex information. Following LD principles, objects are identified with a URI and this URI can be associated to other resources about the object. For example, Al-Khalifa and Hend [21] associate physical objects to their audio description and tag the object with a QR code that contains the URI of its audio description on the Web. This simple cyber-physical object may be used to face accessibility problems of physical objects. It can support visually impaired and blind people to identify objects in the environment.

Our approach uses the architecture for object annotations described in [13]. It is a three-layered architecture consisting of a physical layer, the layer of the digital memory DOM, and a Linked Data layer (see the grey layered boxes in Fig. 1).

(i) The physical layer includes: the physical object, the specific modality of interaction with the object, such as pointing, scanning, touching or using a mediation device (e.g., a smartphone), and the modality of identification of the object, such as RFID, QR-Code, Semacode and techniques for visual object recognition.

(ii) The layer of the digital memory DOM contains the description of the object and the way to access it, according to 
the Object Memory Model (OMM) [20]. Basically it is a repository of digital data that is linked through URIs with a physical artefact.

(iii) The Linked Data layer describes the physical object according to the LD principles and should preferably link the related dataset on the Linked Open Data Cloud (LOD). This layer enables the object to link other objects and to be linked by related objects in the Web of Data (WoD), thus offering the possibility to be shared, extended and reused.

A layered architecture for annotations enables to collect all data concerning an object within a unique logical repository and to expose data about it in a flexible fashion. This architecture is made possible by the new scenario of augmented physical objects, where for example, a Linked Data wrapper can be in charge to publish just a subset of the information stored in the object memory of the object. Ding et al. propose the use of LD to link the user preference and subsets of realtime environment data [22].

The adoption of LD provides several advantages: 1) LD practices are designed to foster the possibility of integrating heterogeneous data and reuse them in different ways [17], 2) the LOD contains increasingly mass information that will complement and enrich the smart object annotation, 3) the object description could be enriched by the users, by creating new triple and thus adding knew knowledge that other users may find precious; and 4) Semantic Web technologies allow for a number of reasoning mechanisms that can sustain the adaptation process. Most of this process can be handled by the state of the art OWL ontology reasoners [23].

Based on the framework, a physical object, such as a laboratory tool, can be provided as a smart IoT tool accessible at different levels. A simple and easy-to-be deployed level is providing the instructions according to the framework principles: text instructions (physical layer) are labeled with a QR code or an RF-ID tag associated to an online DOM which contains several kinds of resources, fitting different types of needs. The LD description allows to link this data to related data on LOD: given that the model of this tool may have features that make it similar to other tools, this connection may allow that all objects of a certain model of the tool may have the same instructions, recommendation and adaptation strategies to be downloaded from the web.

It is worth noting that people, as well as artefacts, being real-world "objects", can have their own digital representation, as showed in Fig. 1 (Person). The layered representation of the user is particularly relevant to our approach, since it enables to store knowledge about the user features and needs concerning accessibility at different levels of visibility, and this data can be used to adapt the interaction with the object (described in the next section). Of course, a vital requirement is the respect of the end-users' privacy when accessing their data. Data about accessibility may be extremely sensitive. Hence, if the adaptation module resides in the object, the user should be allowed to share just the minimal part of her/his profile that is necessary for optimizing the interaction. If the adaptation module resides on the user side (e.g., on personal mobile device) these problems are easier to address, since there is no need to directly inform the smart object about the person's needs or disabilities. Privacy requirements depend on a variety of contextual socio-cultural factors and can be analyzed using privacy requirements distillation approach, such as [24].

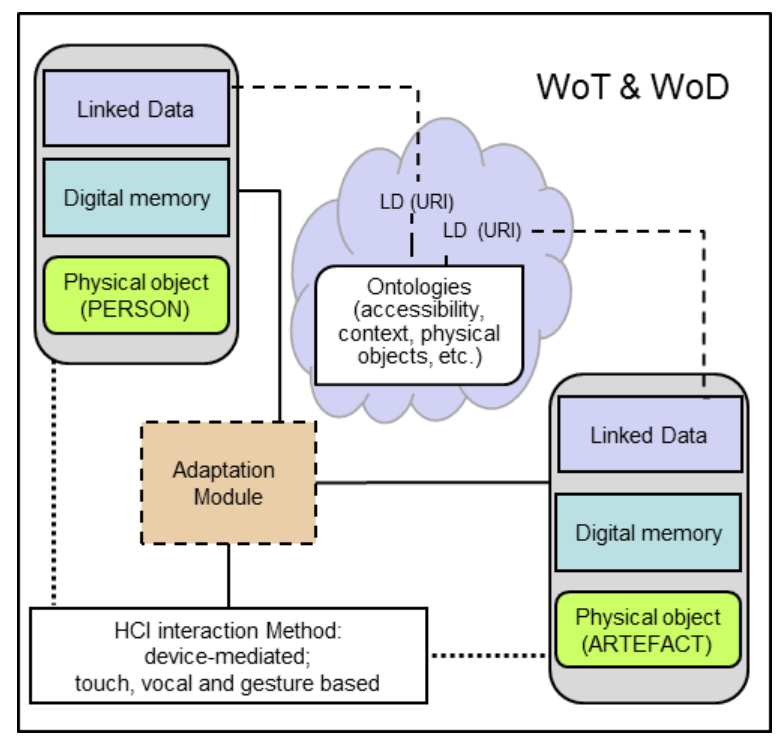

Figure 1. Interaction model of the accessibility framework.

Specific applications implementing the architecture can define how and where to store the digital representation of the objects: locally in the object, or on a server, which can be accessed via HTTP. In such a scenario, virtual representations of objects can serve as central hubs of object information [25] that may combine and continuously update data from a wide range of sources. Part of them can be slowly changing data (e.g. descriptive features of the object), other data may come from sensors and be continuously updated.

Мa [26] proposes a classification that emphasizes this point, proposing a four-layered architecture, including: object sensing layer, data exchange layer, information integration layer, and application service layer. In all the models, the basic idea is that the digital representation of physical objects should harmonize the access to the heterogeneous set of underlying objects with a common language and procedures. This enables applications to get the information they need about the object, based on their specific purpose and users.

\section{Interaction with annotated objects}

Fig. 1 displays the model for the interaction with the augmented physical objects. The object (Artefact) and the user (Person) who wants to access it are represented according to the layered architecture described above. The Linked Data Layer is associated (through a dotted arrow) to the URI in the Linked Data Cloud. This Cloud includes the ontologies that enable the semantic description of the artefacts and of the user features. They provide the vocabularies to represent the RDF statements about the real-world things and accessibility requirements.

Notice that, even though user's data are annotated according to LD principles, they do not necessarily have to be open to public access. Their access can be managed using different polices, depending on the specific implementation of 
the model. Furthermore, the object descriptions can be enriched by the users themselves or supervisors by producing new triples that may be useful to address the need of other users. A number of semantic annotation tools can be used by common users to annotate items (e.g. most CMS include semantic annotation support). This can be quite useful to address the accessibility problem. For example, a user with certain impairment may leave advices of how to best approach a certain item for users with similar disabilities. In the same way, a user speaking in a language that is not supported by the object may decide to help subsequent users by proving a translation of some useful information. Of course, information inserted by users should be handled carefully, since they may include incorrect information. Different policies may thus be implemented to address the trust problem of the different sources. Past experiences, such as semantic wikis [27, 28], proved the potentiality of allowing common users to build their own knowledge.

The bottom left side of the figure shows that the interaction with the object can be mediated by another device, such as a smartphone, or can exploit natural interaction modalities. Mediated access typically uses native applications that get the location of the virtual counterpart of the physical object and access them. Typically, they get the URI of the object and access the Web server at the specified URI. As mentioned above, the server can be embedded within the physical smart object, or on the Web. Natural interaction modalities require specific equipment of the cyber-physical object. For example, the provision of speech modality requires the adoption of ASR (Automatic Speech Recognition) technology and may further implement NLP (Natural Language Processing) techniques; the provision of tactile modality of interaction requires the adoption of multi-touch surface technology. E.g., Microsoft Surface is a development platform that enables to create applications that hide the computer logic below the surface and allow users to interact with a high-end graphics display similar to a coffee table. Thus, users get the service (provided by an application) by using only their fingers.

Finally, Fig. 1 shows the Adaptation Module (AM) that is in charge of adapting the user interface and the interaction modality of the virtual side of the physical objects. It is defined according to the definition of adaptive software [29] and of context-aware adaptive system [30], namely a type of specialized software that uses information from the environment (user needs and context features) to improve its behavior over time. Its function, here, is to use the available information about the object (Artefact) and about the user and its environment (Person) to make the object accessible.

Different configurations are possible for the AM: inside the physical objects, as in distributed models, or externally as in centralized models (on the user's mediation agent or on a web server). A vast literature on adaptive and user modeling systems has been produced in the past [6] about advantages and limits of client side, server side and distributed solutions, however the new scenario of a sensorized society demands for new models.

In general, independently of the architecture, the AM should be in charge of collecting accessibility requirements from different sources, matching them with configuration options of the user interface and user capabilities and adapting the interface accordingly.

In a distributed model, the adaptation is carried out by each physical object, while in a centralized model, a unique module should manage the adaptation for different objects, acting as an agent of the user. In the latter case, given the semantic representation of the user requirements, for example hearing troubles, it should coordinate the different objects so that all of them convert audio notifications into a common haptic feedback, such as a vibration, modulated according to a shared scale of intensity to signify the type of notification, or use tickers or subtitles on displays.

\section{SEMANTIC VIDEO ANNOTATION}

In this section we present a prototypical demonstrator that implements the semantic annotation component described in the framework. The idea is to exploit video annotation in a flipped classroom to support three kinds of impaired students: visually impaired students, hearing impaired students and students affected by learning disorders.

We have identified these types of impaired students as target categories, however it is important to underline that the current demonstrator is not focused on adaptation for different kinds and levels of disability. This will be done in the next step of the design. The current stage just implements the architecture of the framework, that will be used as the basic infrastructure to develop the adaptation service.

This modular approach is made possible by the use of explicit representation of knowledge concerning each component of the framework: the learner model, the real-world objects and the adaptation techniques.

As we mentioned in the Introduction, flipped classroom concerns a teaching paradigm where traditional in-class lessons are replaced by video recorded lectures and in-class activities concern practice exercises, laboratory experiments, document analysis, debate or speech presentation.

Our objective is to exploit the accessibility framework to support the three categories of impaired students mentioned above, both in online activity and in-class activity.

The approach is the following:

- improving the accessibility of online video lectures by exploiting semantic annotations and adapting them to the type of impairment,

- $\quad$ improving the accessibility of in-class activity by:

$\checkmark \quad$ video recording in-class activities

$\checkmark$ annotating them with semantic annotations and adapting them to the type of impairment.

Video lecturers are digital objects therefore they can implement only the second and third layer of the layered architecture for object annotation. Differently, in-class activities are real-world scenes made of real-world things. Therefore we are able to implement all of the theoretical layers of the accessibility framework. 
In order to manage the semantic annotation we exploit Apache Marmotta platform.

\section{A. Platform for video annotation}

The current implementation is the integration of a responsive interface and an annotation tool, both realized using HTML5-CSS3-JS technologies like the Foundation framework from Zurb ${ }^{1}$, built on top of a Linked Data Endpoint service using Apache Marmotta 3.32.

Apache Marmotta is a top level project of Apache Software Foundation, and provides an open source Linked Data Platform for the interlinking of data repositories with the Web of Data according to the principles of the World Wide Web Consortium (W3C). It has been developed as a continuation and simplification of the Linked Media Framework (LMF) project ${ }^{3}$. With LMF framework, the research group in Salzburg extended the LD principles to multimedia content (videos, photos, graphics, etc.), while at the same time realizing $\mathrm{W} 3 \mathrm{C}$ 's vision of the Read-Write-Web. Marmotta is highly modular and extensible to build custom LD applications, like our prototype. Its core components are the Linked Data Server with SPARQL 1.1. and the LD Cache.

A useful option in Marmotta is the extension of SPARQL with specific multimedia functions and relations (e.g., rightBeside, spatialOverlaps, after, etc. ).

For the annotation of video contents, we followed the principles in [30]. Video resources are URI, and locally we add annotations (tags) to denote video fragments (parts of the original URI) creating RDF triples which encode temporal and spatial subsequences building semantic relationships between an object/human/artefact and the video fragment.

The semantic annotation tool, displayed in Fig. 2, allows teachers and authors of educational resources to search videos on large social repositories (Youtube and Vimeo), as well as on social networks (Facebook), and connecting social tagging with formal/informal classifications (Wikipedia, WikiCommons) by means of Linked Data. The semantic Linked Data Endpoint is managed as an instance of Apache Marmotta.

\section{B. Layered semantic video annotation}

In this section we analyze in-class activities given that they fully fit the scope and requirements of the accessibility framework. The goal is to make "in-class scenes" understandable to students with different types of disability.

Based on the accessibility framework, the first action to be performed is enriching physical objects with semantic annotations. In this instantiation of the framework, the realworld objects are the in-class activities. This represents a very complex object since an in-class activity is composed of nested real-world objects that have to be managed independently. According to the layered architecture for object annotations, the digital memory of the object (DOM) is a repository of digital data that is linked with a physical object, and may be

\footnotetext{
${ }^{1}$ http://foundation.zurb.com/

2 http://marmotta.apache.org

${ }^{3}$ http://www.w3.org/2001/sw/wiki/LMF
}

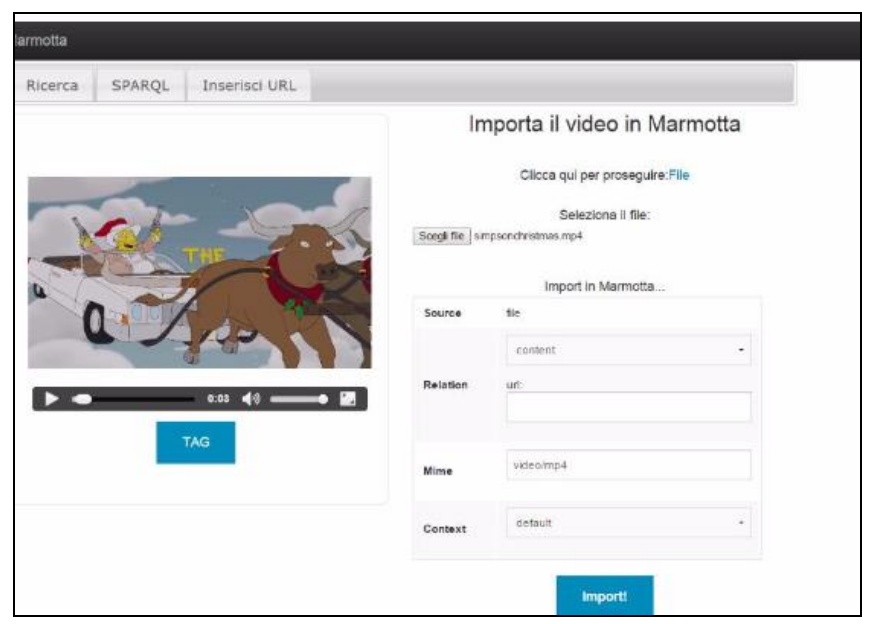

Figure 2. The semantic annotation tool interface for importing a video.

populated with static or dynamic data from entities that interact virtually or physically with the artefact.

Since we have scenes composed of things, we need first to create a DOM of each object. Video recording and pictures of the object will form the set of materials associated to the virtual representation of the physical object.

The approach we adopted is described below:

- to record the scenes of the in-class activity,

- to capture the real-world things in the class (in order to populate a database for pattern recognition and annotation); this includes capturing both animated things (persons) and unanimated things (artefacts),

- to identify automatically or semi-automatically things in the scenes, creating and annotating fragments (on the timeline ),

- manually and collaboratively annotating actions concerning the in-class activity.

According to the architecture for object annotation, the third layer is the Linked Data layer, defined as a subset of DOM. Each object and video is annotated using LD and stored in Marmotta as RDF Triples. In the previous sections we extensively discussed the flexibility of a layered architecture and the reasons for using the LD approach.

In order to enhance interoperability and interlinking in the LOD, it is useful to annotate objects by using popular and effective ontologies such as the following ones:

- $\mathrm{FOAF}^{4}$ is an ontology for person annotation;

- ACCESSIBLE $^{5}$ and AEGIS ${ }^{6}$ are ontologies for accessibility related features (their integration provides a multilayer ontology which includes standards, guidelines, techniques and the description of device features, functional limitations of users with disabilities and impairments. Moreover ACCESSIBLE includes verification rules for describing accessibility requirements and constraints);

\footnotetext{
${ }^{4}$ http://www.foaf-project.org/

${ }^{5}$ http://160.40.50.89/Accessible_Ontology

${ }^{6}$ http://160.40.50.89/AEGIS_Ontology
} 
- Ontology for Media Resources ${ }^{7}$ is both a core vocabulary (a set of properties describing media resources) and its mapping to a set of metadata formats currently describing media resources published on the Web. It is a W3C Recommendation.

- $\quad$ Other domain-specific or multi-domain ontologies may be necessary to annotate things. In our demonstrator we have exploited DBpedia to add tags to objects in recorded inclass scenes and also to add tags to objects and persons in video lecturers (see Fig. 3).

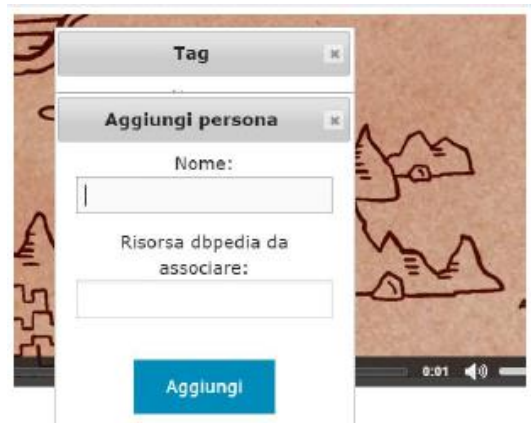

Figure 3. The tool interface for adding a person linked to a DBpedia resource.

Depending on the kind of disability, different types of annotations have to be included. For visually impaired students, audio recording has to be synchronized with precise descriptions of the objects and of the actions. For hearing impaired students, annotations have to transcript audio recording, managing overlapping voices and noise. Finally, for students with learning disorders it could be necessary to provide a different type of annotation, which enables students to understand the scene. For example by using analogy-based methods or by retrieving linked concepts ${ }^{8}$.

To manage the last case, we have performed some experimental tests by retrieving DBpedia data linked to the objects in the scenes stored in Marmotta. The result is that the annotation is driven by some recommendations that are automatically generated and saved in the LD Cache of Marmotta.

Thus, new knowledge is created from both pattern recognition on captured video fragments (keyframe and objects identification) and knowledge retrieval from the LOD cloud. The former is referred to as intensional knowledge and the latter as extensional knowledge.

Notice that the tool for semantic video annotation is part of the accessibility framework but it can be usefully exploited with non-impaired students as well. In fact, despite a huge amount of recorded material is available in video repositories, a detailed description of their contents is lacking. Such videos could include meaningful notions and show examples as well as standard situations, use cases or case histories. To make

\footnotetext{
${ }^{7}$ http://www.w3.org/TR/mediaont-10/

${ }^{8}$ Several researches on learning disorders are available such as [32]. Our demonstrator is inspired to this work but does not implement its methods strictly since our current objective is to set up the knowledge base infrastructure and investigate the validity of the approach.
}

them searchable and usable, the relevant frames should be identified and tagged. The platform for video annotation that we presented above is an easy-to use and powerful tool that can be used by experts but also by students as we experimentally tested in our setup.

About this, it is worth observing that a way to include students with different needs is to provide them a role which enhances their abilities [32]. Collaborative tagging could be an effective instructional activity that follows the principles of knowledge building and constructivism, but could be also an effective strategy to produce different types of annotations for students with different needs.

\section{CONCLUSION}

In this paper we have presented a framework for exploiting Web of Things, semantic annotation and adaptation techniques to support impaired people to access real-world things. Subsequently, based on this framework, we have discussed the implementation of a video annotation set up that is the basic infrastructure for increasing accessibility to in-class activities and online lecturers. The prototypical implementation presented in the paper addresses the knowledge base requirements for video annotation.

The contribution of the paper is twofold: (i) from a theoretical point of view it presents a general-purpose innovative model for enhancing the accessibility to things that are in the physical world (e.g., books, calculators, laboratory tools, etc.) or that happen in the physical world (e.g., in-class activities), showing the potential power of LD-based annotation; (ii) from an educational point of view, the approach of video recording and semantic annotating in-class activities fits the scenario of flipped classrooms, and proposes a novel approach to face accessibility problems of impaired students.

As a future work a deep study on sets of disabilities and of associated requirements and adaptation techniques is planned.

\section{ACKNOWLEDGMENTS}

This research has been funded by the University of Genoa, within PRA 2013 projects, Prot. N. 9563.

\section{REFERENCES}

1. Domingo, M., C., 2012. An overview of the Internet of Things for people with disabilities, Journal of Network and Computer Applications, Vol. 35, pp. 584-596.

2. O'Flaherty, J., Phillips, P., 2015 The use of flipped classrooms in higher education: A scoping review, The Internet and Higher Education, Vol. 25, April 2015, pp. 85-95.

3. Aubert, O. and Jaeger, J. Annotating Video with Open Educational Resources in a Flipped Classroom Scenario. CoRR abs/1412.1780, 2014.

4. Guo, P. J., Kim, J., \& Rubin, R., How video production affects student engagement: An empirical study of mooc videos. In Proceedings of the first ACM conference on Learning@scale conference, 2014,pp.41-50. 
5. Risko, E. F. , T. Foulsham, S. Dawson, A. Kingstone, The Collaborative Lecture Annotation System (CLAS): A New TOOL for Distributed Learning", IEEE Transactions on Learning Technologies, vol.6, no. 1, 2013 pp. 4-13.

6. Pardo, A., Mirriahi, N., Dawson, S., Zhao, Y., Zhao, A., \& Gašević, D. Identifying learning strategies associated with active use of video annotation software. In Proc. of the Conference on Learning Analytics And Knowledge, 2015, pp. 255-259.

7. Encelle, B., Ollagnier-Beldame, M., Pouchot, S., \& Prié, Y. Annotation-based video enrichment for blind people: A pilot study on the use of earcons and speech synthesis. In Proc. of the ACM Conference on Computers and accessibility, 2011, pp.123-130.

8. Clarkson, P.J., Coleman, R., Keates, S., Lebbon, C. Inclusive design: Design for the whole population. Springer Science \& Business Media, 2003.

9. Stephanidis, C. and Antona, M. (eds), Universal Access in Human-Computer Interaction. LNCS, Vol. 8513, 2014.

10. Fortino, G., Russo, W., Rovella, A., Savaglio, C. On the Classification of Cyberphysical Smart Objects in Internet of Things. Proc. of the Int. Workshop on Networks of Cooperating Objects for Smart Cities (UBICITEC). Vol. 1156, 2014, pp. 76-84.

11. Lee, A. E. Cyber Physical Systems: Design Challenges. In Proc. of the 11th IEEE Symposium on Object Oriented Real-Time Distributed Computing IEEE Computer Society, Washington, DC, USA, 2008, pp. 363-369.

12. Featherstone, D. An Inclusive Internet of Things: Accessibility in the Palm of Your Hand, available at http://simplyaccessible.com/article/things/, accessed 18 April, 2014.

13. Torre, I. Interaction with Linked Digital Memories. Proc. of the Int. Workshop on Personalized Access to Cultural Heritage within UMAP, Rome, July 14, CEUR Proc, vol. 997, 2013, pp. 80-87.

14. Torre, I. Virtualization of Objects and Adaptive Interaction in an Inclusive Web of Things, Proc. of the Int. Conference on Interfaces and Human Computer Interaction, pp. 15 - 17 July 2014, Lisbon, Portugal.

15. Coccoli, M., Torre, I., Interacting with annotated objects in a semantic web of things application, Journal of Visual Languages and Computing, Volume 25, Issue 6, 2014, pp. 1012-1020.

16. Adorni, G., Coccoli, M., Torre, I. Journal of E-Learning and Knowledge Society, Volume 8, Issue 2, May 2012, pp. 23-32.

17. Heath, T. and Bizer, C., Linked Data: Evolving the Web into a Global Data Space. Lectures on the Semantic Web, Morgan \& Claypool, 2011, pp. 1-136.

18. Zhou, L., Parker, A. T., Smith, D. W., \& Griffin-Shirley, $\mathrm{N}$. Assistive technology for students with visual impairments: Challenges and needs in teachers' preparation programs and practice. Journal of Visual Impairment \& Blindness, 105(4), 2011, pp. 197-210.

19. Kaklanis, N., Votis, K., Giannoutakis, K., and Tzovaras, D. A Semantic Framework for Assistive Technologies Description to Strengthen UI Adaptation, Universal Access in HCI, LNCS Vol. 8513, 2014, pp 236-245.

20. Barthel, R., Kröner, A., Haupert, J. Mobile interactions with digital object memories, Pervasive and Mobile Computing, Vol. 9(2), 2013. pp. 281-294.

21. Al-Khalifa, Hend S. Utilizing QR code and mobile phones for blinds and visually impaired people. LNCS Vol. 5105, 2008, pp 1065-1069.

22. Ding, C., Wald, M. and Wills, G. Linked data for accessibility: from techniques to users. Proc. Int. Conf. on e-Society, Lisbon, PT, 13 - 16 Mar 2013, pp. 514-516.

23. Sirin, E., Parsia, B., Grau, B. C., Kalyanpur, A., \& Katz, Y. Pellet: A practical owl-dl reasoner. Web Semantics: science, services and agents on the World Wide Web, 5(2), 2007, pp. 51-53.

24. Thomas, K., Bandara, A. K., Price, B. A., \& Nuseibeh, B. Distilling privacy requirements for mobile applications. In Proc. of the ACM Conference on Software Engineering , 2014, pp. 871-882.

25. Verdouw, C. N,. Beulens, A. J. M and Vorst van der J. G. A. Virtual Logistic Networks in Dutch Horticulture. Proc. of the 4th Production and Operations Management World Conference, Amsterdam, 2-4 July, 2012.

26. Ma, H.D., Internet of Things: Objectives and Scientific Challenges. Journal of Computer Science and Technology. Vol. 26, No 6, 2011, pp. 919-924.

27. Coccoli, M., Vercelli, G., Vivanet, G., Semantic Wiki: A collaborative tool for instructional content design, Journal of E-Learning and Knowledge Society, Volume 8, Issue 2, May 2012, pp. 113-122.

28. Krötzsch, M., Vrandečić, D., \& Völkel, M. Semantic mediawiki. In The Semantic Web-ISW, Springer Berlin Heidelberg, 2006, pp. 935-942.

29. Norvig, P. and Cohn, D,. Adaptive software. PC AI Magazine, Vol. 11, No. 1, 1997, pp. 27-30.

30. Dey, A.K., Abowd, G.D. and Salber, D. A conceptual framework and a toolkit for supporting rapid prototyping of context-aware applications, Human-Computer Interactions Journal, Vol. 16(2), 2001, pp.97-166.

31. Kurz, T., Güntner, G., Damjanovic, V., Schaffert S. and Fernandez, M. Semantic enhancement for media asset management systems. Multimedia Tools Appl. 70(2), 2014, pp. 949-975.

32. Reid, Robert, Torri Ortiz Lienemann, and Jessica L. Hagaman. Strategy instruction for students with learning disabilities. Guilford Publications, 2013. 